\title{
Assessment of N-acetyltransferase 2 (NAT2) gene polymorphisms in bladder cancer patients
}

\author{
Sayeda Abd El Rahiem Saleh ${ }^{1 \oplus}$, Heba M Adel Abou Zaghla $^{1^{\oplus}}$, Somia Abdel Hamid Bawady ${ }^{{ }^{*}}{ }^{\circledR}$, \\ Mohamed Kotb $^{2}{ }^{\mathbb{D}}$, Walaa Ibrahim Hammad ${ }^{1}$ \\ ${ }^{1}$ Clinical Pathology Department, Faculty of Medicine, Ain-Shams University, Cairo, Egypt \\ ${ }^{2}$ Urology Department, Faculty of Medicine, Ain-Shams University, Cairo, Egypt
}

\section{A R T I CLE I N F O}

Article Type:

Original

\section{Article History:}

Received: 10 January 2019

Accepted: 3 May 2019

Published online: 10 June 2019

\section{Keywords:}

Bladder cancer, gene polymorphisms, Carcinogenic toxins, Slow acetylator phenotype, $\mathrm{N}$-acetyltransferases phenotypes, Cigarette

\begin{abstract}
A B S T R A C T
Introduction: Bladder cancer is the most common cancer of the urinary tract. Aromatic amines are incriminated in the pathogenesis of bladder cancer. While many people are exposed to it, only a small proportion of the exposed individuals develop bladder cancer indicating the contribution of genetic variation, namely $\mathrm{N}$-acetyltransferases (NATs) phenotypes (slow and fast acetylator) which are involved in detoxification of aromatic amines and other carcinogenic toxins. Slow acetylators are less efficient in metabolizing aromatic amines, increased toxic burden and subsequently carcinogenesis.

Objectives: The aim of this study was to determine whether NAT2 gene polymorphisms (M1, M2 and M3) are associated with bladder cancer formation and with high stage and grade of the tumor. Patients and Methods: This study was conducted on 35 Egyptian bladder cancer patients. Patients further subdivided according to tumor stage and grade. Fifteen patients with benign renal diseases as a pathological control group, in addition to 15 apparently healthy subjects as a healthy control group included in the study. Assay of NAT2 gene single nucleotide polymorphisms (SNPs) was conducted by polymerase chain reaction and restriction fragment length polymorphism (PCRRFLP) technique.

Results: Regarding the frequency of NAT2 gene polymorphism, our study revealed that M1 (C481T) mutation was significantly more frequent in patients than controls groups, however no significant differences with M2 or M3 was seen.

Conclusion: This investigation implies that NAT2 genotype exhibit no association with the risk of developing bladder cancer, either alone or with smoking. Moreover, there was no association between NAT2 polymorphism and different stages and grades of bladder cancer.
\end{abstract}

\section{Implication for health policy/practice/research/medical education:}

In a study on 35 Egyptian bladder cancer patients, we found that N-acetyltransferase 2 (NAT2) genotype exhibit no relationship with the risk of developing bladder cancer, either alone or with smoking.

Please cite this paper as: El Rahiem Saleh SA, Abou Zaghla HMA, Abdel Hamid Bawady S, Mohamed Kotb M, Hammad W. Assessment of N-acetyltransferase 2 (NAT2) gene polymorphisms in bladder cancer patients. J Nephropharmacol. 2019;8(2):e30. DOI: $10.15171 /$ npj.2019.30

\section{Introduction}

Bladder cancer is the most common cancer of the urinary tract, accounting for approximately 330000 new cases and 130000 deaths per year worldwide (1). It is the seventh most common cancer in men and the 17 th most common cancer in women worldwide (2). In Egypt it accounts for $17 \%$ of all cancer cases in males, while in females it accounts for 5\% (3). It is now accepted that the cause of bladder cancer is a multifactorial interaction between environmental factors and genetic susceptibility (4).

The risk factors for bladder cancer include exposure to arylamines in occupational settings or from cigarette smoke (2).

The gold standard for bladder cancer screening is urine cytology, as it is non-invasive, safe and inexpensive. Although it is highly specific, the results are not reproducible and the interpretation is highly dependent on the skill of the cytologist (3). Although conventional cystoscopy is considered to be the gold standard for diagnosis and follow-up of bladder tumors, it remains an invasive and costly procedure (5). This problem calls for searching other markers for screening of bladder cancer 
which should be specific, sensitive, reproducible, and non-invasive with an acceptable cost (3). Although many people are exposed to environmental risk factors, only a small proportion of the exposed individuals develop bladder cancer, indicating that, other factors, namely genetic variation might contribute to the development and progression of bladder cancer. Human $\mathrm{N}$-acetyltransferases (NATs) are involved in the metabolism of drugs, environmental toxins and aromatic amine carcinogens. NATs catalyze the transfer of an acetyl group from acetyl-CoA to the nitrogen or oxygen atom of arylamines, hydrazines and their N-hydroxylated metabolites hence converting them to inactive compounds. There are two functional human NATs; NAT1 and NAT2. The NAT2 gene is polymorphic and is located on chromosome 8 p22. These single nucleotide polymorphisms (SNPs) affect the acetylation rate. The activity of NAT2 is predicted from the combination of the NAT2 alleles. The presence of at least one wild type allele is considered a fast acetylator where the absence of any wild type allele is considered as slow acetylator (6). The two NAT2 phenotypes have been described; fast and slow. Slow acetylators are less efficient in metabolizing aromatic amines, increased toxic burden and ultimately carcinogenic hallmarks such as DNA adduct formation and oxidative stress and hence may increase the risk of cancer (7).

\section{Objectives}

The aim of this study is to study the relationship between NAT2 gene polymorphism and bladder cancer and also to assess its correlation with different histopathological stages and grades of the disease using polymerase chain reaction and restriction fragment length polymorphism (PCR-RFLP).

\section{Patients and Methods}

\section{Study population}

Our study was conducted on 35 Egyptian bladder cancer patients (group I, mean age was $55 \pm 7.6$ years) who referred to urology department from January 2017 to August 2017, at Ain Shams University hospitals in Egypt and diagnosed by histopathological examination. Group II included 15 patients (mean age of $47 \pm 7.6$ years) with benign renal diseases such as renal stones, bladder stones and cystitis served as a pathological control group. In addition to the group III which included 15 apparently healthy subjects (mean age was $51.7 \pm 9.3$ years) served as a healthy control group. Assay of NAT2 gene SNPs was conducted by PCR-RFLP technique. The study was done after approval of the ethical board of Ain-Shams University. Informed consent was taken from each participant in the study. All individuals included in this study were subjected to full history taking and clinical examination with special emphasis smoking, radiological examinations including ultrasonography and computed tomography for patient and pathological control groups, urine cytology, cystoscopy and bladder biopsy (for patient group only) and assay of NAT 2 gene polymorphism by PCR-RFLP analysis in the peripheral blood.

Genotyping of NAT2 gene polymorphisms gene polymorphism was conducted in peripheral blood using PCR-RFLP technique. Genomic DNA was extracted from EDTA- anticoagulated peripheral whole blood by a GeneJET DNA purification kit supplied by Thermo Scientific (168 Third Avenue, Waltham, MA, USA). Amplification of extracted DNA was done using master mix supplied by Thermo Scientific (168 Third Avenue, Waltham, MA, USA), which contains chemically modified Maxima hot start Taq DNA polymerase, optimized hot start PCR buffer, $\mathrm{Mg}^{+2}$ and dNTPs (deoxynucleotide triphosphates), and the primers supplied by Invitrogen (5791 Van Allen Wa, Carlsbad, CA, USA) with the following sequence: forward 5"-TCT AGC ATG AAT CAC TCT GC -“3 \&reverse 5”-GGA ACA AAT TGG ACT TGG - "3. PCR amplification started with initial activation of hot start Taq DNA polymerase for 15 minutes at $95^{\circ} \mathrm{C}$, followed by 35 cycles $\left(95^{\circ} \mathrm{C}\right.$ for 1 minute for DNA denaturation, $55^{\circ} \mathrm{C}$ for 1 minute for annealing and extension at $72{ }^{\circ} \mathrm{C}$ for 1 minute) and finished by a final extension step at $72^{\circ} \mathrm{C}$ for 10 minutes. The PCR reaction mixture (total volume $50 \mu \mathrm{L}$ ) contained $20 \mu \mathrm{L}$ of DNA template, $2 \mu \mathrm{L}$ of each primer and $25 \mu \mathrm{L}$ of master mix, then molecular-grade $\mathrm{H} 2 \mathrm{O}$ was added at the end to adjust the total volume of the PCR reaction mixture to $50 \mu \mathrm{L}$. The 1092 bp PCR amplified products were digested with three restriction enzymes separately which are Klebsiella pneumonia (kpnI) specific for C481T polymorphism (M1), Thermus aquaticu (Taq1) specific for G590A (M2) polymorphism and Bacillus amyloliquefaciens (BamHI) specific for G857A (M3) polymorphism. Digestion by restriction enzymes was done for 15 minutes at $37^{\circ} \mathrm{C}$ in case of BamHI and $\mathrm{KpnI}$ while at $65^{\circ} \mathrm{C}$ in case of Taq1. Digested products were analysed by electrophoresis on $2 \%$ agarose gel stained with ethidium bromide for 20 minutes at 100 volts. A DNA molecular weight marker was also run in each gel to identify the site of bands (50 bp ladder) which were visualized by ultraviolet trans-illumination. KpnI restriction enzyme recognizes $\mathrm{GGTAC}^{\wedge} \mathrm{C}(\mathrm{M} 1)$ site. If $\mathrm{C}$ is substituted by $\mathrm{T}$ at position 481 , then $\mathrm{KpnI}$ restriction site will be lost and the bands will be one of the following; homozygous mutation with only one band develops at $1092 \mathrm{bp}$, heterozygous mutation with the three bands develop at 1092 bp, 648 bp and 444bp and Wild type: two bands develop at $648 \mathrm{bp}$ and $444 \mathrm{bp}$ as shown in Figure 1.

Taq I restriction enzyme recognizes $\mathrm{G} \wedge \mathrm{A}$ sites. There are TaqI sites (two of three Taq I sites) common to all NAT2 polymorphic alleles. When $\mathrm{G}$ is replaced by $\mathrm{A}$ at position 590 , one TaqI site will be lost and the bands will be one of the following ways; homozygous mutation 3 bands develop at 396, 370, 326, heterozygous mutation with 5 bands develop at 396, 370, 326, 226, 170 and wild type with 4 bands develop at $370,326,226,170$ as shown in 
Figure 2.

BamHI recognizes site at $G^{\wedge} G(M 3)$. If $G$ is replaced by $A$ at position 857, then BamHI restriction site will be lost and the bands will be one of the following ways; homozygous mutation only one band develops at $1092 \mathrm{bp}$, heterozygous mutation with three bands develop at $1092 \mathrm{bp}, 820 \mathrm{bp}$ and $272 \mathrm{bp}$ and wild type with two bands develop at $820 \mathrm{bp}$ and 272 bp as shown in Figure 3.

\section{Ethical approval}

The study was in accordance with the Declaration of Helsinki and its later amendments. All participants gave their informed consent to enter the study. The study has been approved by the ethical committee of Faculty of Medicine, Ain-Shams University.

\section{Statistical analysis}

The data were coded, entered and analysed using Statistical Package for SPSS version 22.0 (IBM Corp., USA, 2013). Data were statistically described in terms of, mean \pm SD (standard deviation) for quantitative parametric data, median and interquartile range for quantitative nonparametric data and percentage for qualitative data. For comparing categorical data, chi-square test was performed. A probability value ( $P$ value) more than 0.05

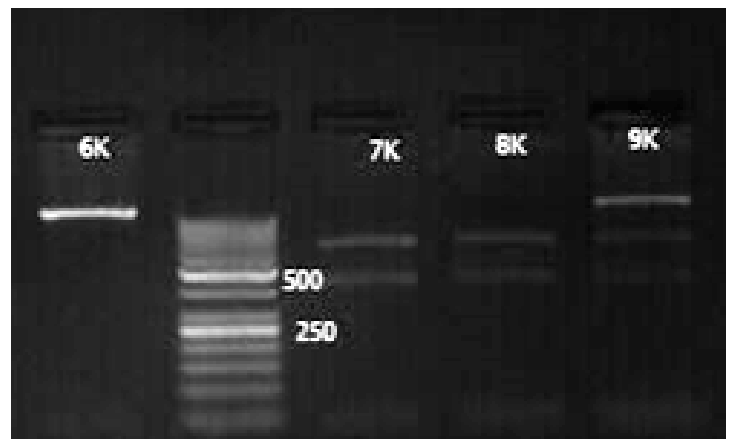

Figure 1. PCR-RFLP results by $\mathrm{Kpnl}(\mathrm{K}) .6 \mathrm{k}$ is mutant type, 7 and $8 \mathrm{~K}$ are wild types, and $9 \mathrm{~K}$ is a heterozygous allele.

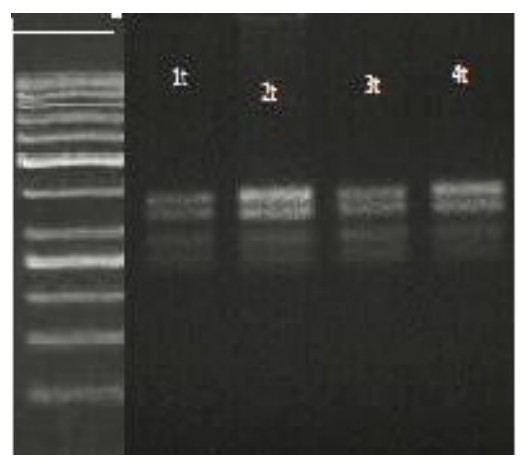

Figure 2. PCR-RFLP results by Taq1 (T). 1, 2, 3 and 4 T are wild type.

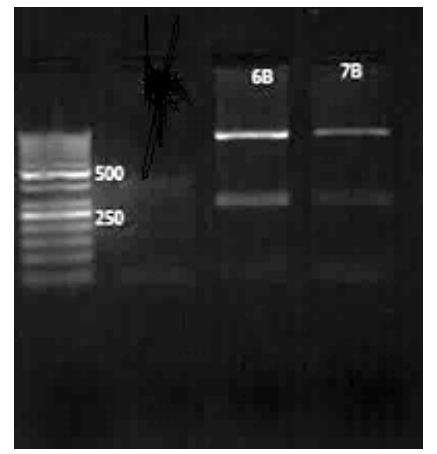

Figure 3. PCR-RFLP results by BAMHI (B). 6B and 7 B are wild types.

was considered non statistically significant.

Results

The results of this study are presented in Tables 1 to 5 . Table 2 shows the descriptive and comparative statistics of the genotypes frequencies of the studied NAT2 SNPs in bladder cancer patients (group I), pathological controls (group II) and healthy controls (group III). It revealed a statistical significant difference between the different genotypes frequencies of NAT2 gene polymorphism on restriction with KpnI for C481T SNP (M1) between the different studied groups $\left(\chi^{2}=13.34, P<0.05\right)$. However, non-statistical significant difference regarding NAT2 acetylation status among different stages and grades of bladder cancer patients was detected $\left(\left(\chi^{2}=3.54\right.\right.$, $0.82, P>0.05$; respectively). Statistical comparison was conducted between NAT2 acetylation status and smoking in bladder cancer (group I), pathological controls (group II) and healthy controls (group III). It revealed a nonstatistical significant difference between the different studied groups $\left(\left(\chi^{2}=0.81, P>0.05\right)\right.$.

Discussion

Bladder cancer is the ninth most common cancer throughout the world. More than 12 million new cases of cancer occur annually worldwide (8).

Table 1. Descriptive statistics of the demographic and laboratory data in bladder cancer patients (group 1), pathological controls (group 2) and healthy controls (group 3)

\begin{tabular}{lccc}
\hline Parameter & $\begin{array}{c}\text { Group 1 } \\
(\mathbf{n = 3 5 )}\end{array}$ & $\begin{array}{c}\text { Group 2 } \\
(\mathbf{n = 1 5 )}\end{array}$ & $\begin{array}{c}\text { Group 3 } \\
(\mathbf{n = 1 5 )}\end{array}$ \\
\hline Age (years) & $(55 \pm 7.6)$ & $(47 \pm 7.6)$ & $(51.7 \pm 9.3)$ \\
Sex $\quad$ Male & $32(91.4 \%)$ & $13(86.7 \%)$ & $12(80 \%)$ \\
$\quad$ Female & $3(8.6 \%)$ & $2(13.3 \%)$ & $3(20 \%)$ \\
Smokers & $30(85.7 \%)$ & $11(73.3 \%)$ & $12(80 \%)$ \\
Macroscopic hematuria & $28(80 \%)$ & $2(13.3 \%)$ & $0(0 \%)$ \\
Microscopic hematuria & $32(91 \%)$ & $8(53 \%)$ & $0(0 \%)$ \\
Pyuria & $17(48.6 \%)$ & $6(40 \%)$ & $0(0 \%)$ \\
Positive cytology & $20(57 \%)$ & $0(0 \%)$ & $0(0 \%)$ \\
\hline
\end{tabular}


Table 2. Descriptive and comparative statistics of the genotypes frequencies of the studied NAT2 gene SNPs in bladder cancer patients (group 1), pathological controls (group 2) and healthy controls (group 3) using chi-square test

\begin{tabular}{|c|c|c|c|c|c|}
\hline Genotype & Group $1 \quad(n=35)$ & Group 2 ( $n=15)$ & Group $3 \quad(n=15)$ & $\chi^{2}$ & $P$ \\
\hline \multicolumn{6}{|l|}{ M1/C481T } \\
\hline $\mathrm{CT}$ & $1(2.9 \%)$ & $3(20 \%)$ & $1(6.7 \%)$ & \multirow{3}{*}{13.34} & \multirow{3}{*}{$<0.05$} \\
\hline $\mathrm{TT}$ & $12(34.3 \%)$ & $1(6.7 \%)$ & $0(0 \%)$ & & \\
\hline $\mathrm{CC}$ & $22(62.9 \%)$ & $11(73.3 \%)$ & $14(93.3 \%)$ & & \\
\hline \multicolumn{6}{|l|}{ M2/G590A } \\
\hline GA & $2(5.7 \%)$ & $1(6.7 \%)$ & $1(6.7 \%)$ & \multirow{3}{*}{0.63} & \multirow{3}{*}{$>0.05$} \\
\hline AA & $1(2.9 \%$ & $1(6.7 \%)$ & $1(6.7 \%)$ & & \\
\hline GG & 32 (91.4\%) & $13(86.7 \%)$ & $13(86.7 \%)$ & & \\
\hline \multicolumn{6}{|l|}{ M3/G857A } \\
\hline GA & $3(8.6 \%)$ & $2(13.3 \%)$ & $0(0 \%)$ & \multirow{3}{*}{3.25} & \multirow{3}{*}{$>0.05$} \\
\hline AA & $1(2.9 \%)$ & $1(6.7 \%)$ & $0(0 \%)$ & & \\
\hline GG & 31 (88.6\%) & $12(80 \%)$ & $15(100 \%)$ & & \\
\hline
\end{tabular}

Table 3. Statistical comparison of NAT2 gene polymorphism (M1, M2 and M3) among different pathological stages of bladder cancer patients using chisquare test

\begin{tabular}{|c|c|c|c|c|c|}
\hline Genotype & Stage $1(n=17)$ & Stage $2(n=11)$ & Stage $3(n=7)$ & $\chi^{2}$ & $P$ \\
\hline M1/C481T & & & & 4.93 & $>0.05$ \\
\hline $\mathrm{CT}$ & $0(0 \%)$ & $6(54.5 \%)$ & $1(14.3 \%)$ & & \\
\hline $\mathrm{TT}$ & $6(35.3 \%)$ & 2 (18.1\%) & $3(42.9 \%)$ & & \\
\hline $\mathrm{CC}$ & $11(64.7 \%)$ & $3(27.4 \%)$ & $3(42.9 \%)$ & & \\
\hline \multicolumn{6}{|l|}{ M2/G590A } \\
\hline GA & $1(5.9 \%)$ & $0(0 \%)$ & $1(14.3 \%)$ & \multirow{3}{*}{3.80} & \multirow{3}{*}{$>0.05$} \\
\hline AA & $0(0 \%)$ & $1(9.1 \%)$ & $0(0 \%)$ & & \\
\hline GG & $16(94.1 \%)$ & $10(90.9 \%)$ & $6(85.7 \%)$ & & \\
\hline \multicolumn{6}{|l|}{ M3/G857A } \\
\hline GA & $3(17.6 \%)$ & $0(0 \%)$ & $0(0 \%)$ & \multirow{3}{*}{4.82} & \multirow{3}{*}{$>0.05$} \\
\hline AA & $1(5.9 \%)$ & $0(0 \%)$ & $0(0 \%)$ & & \\
\hline GG & $13(76.5 \%)$ & 11 (100\%) & $7(100 \%)$ & & \\
\hline
\end{tabular}

NATs are phase II metabolic enzymes. They catalyse the acetylation of aromatic and heterocyclic amine carcinogens (9). Their activity is encoded by 2 distinct genes, named NAT1 and NAT2. NAT2 gene has been reported to exhibit a polymorphism. Subsequently, the presence of the different alleles in each individual genome produces a broad range of metabolic phenotypes that vary from fully active rapid metabolizers to the less active alleles of slow metabolizers (3). There are remarkable genetic polymorphisms of NAT2 activity have been associated with different levels of susceptibility to develop many kinds of cancers (10).

Regarding the frequency of NAT2 gene polymorphism, our study revealed a significant difference of M1 mutation between the different studied groups. On comparing the groups with each other, it revealed that M1 mutation was significantly more frequent in bladder cancer patients. Our results are in accordance with the findings of Hosen et al (1) who reported that M1 was detected in $49 \%$ of patients and only $20 \%$ of controls.

On the other hand, there were no significant differences neither in M2 nor M3 among bladder cancer patients and control groups. These data are in accordance to Vilčková et al (6) who reported that M2 was detected in $8.89 \%$ of bladder cancer patients and $8.38 \%$ of controls, while M3 was detected in $2 \%$ in both patients and controls.

In this study, it was found that 32 out of 35 bladder cancer individuals and 14 out of 15 subjects of each control group were identified as fast acetylators. Consequently, on comparing the frequency of NAT2 slow and fast acetylation genotype in the different studied groups, it revealed, no statistical significant difference. Our results are consistent with the study of Mittal et al (11) which were done to show whether NAT2 genotypes and smoking are risk factors for bladder cancer in a group of patients from rural India. Their study included 110 controls and 101 bladder cancer cases diagnosed as transitional cell carcinoma of different grades. Results showed that there was no statistically significant difference between bladder cancer and controls as regards fast and slow acetylation genotypes.

The same results were concluded in a study done 
Table 4. Statistical comparison of NAT2 gene polymorphism (M1, M2 and M3) among different grades of bladder cancer patients using chi-square test

\begin{tabular}{lcccc}
\hline Genotype & $\begin{array}{c}\text { Grade I } \\
(\mathbf{n = 2 8})\end{array}$ & $\begin{array}{c}\text { Grade II } \\
(\mathbf{n}=\mathbf{7})\end{array}$ & $\boldsymbol{\chi}^{2}$ & $\boldsymbol{P}$ \\
\hline M1/C481T & $0(0 \%)$ & $1(14.3 \%)$ & & \\
CT & $10(35.7 \%)$ & $2(28.6 \%)$ & & $>0.13$ \\
TT & $18(64.3 \%)$ & $4(57.1 \%)$ & & \\
CC & $1(3.6 \%)$ & $1(14.3 \%)$ & & \\
M2 /G590A & $1(3.6 \%)$ & $0(0 \%)$ & & $>0.05$ \\
GA & $26(92.9 \%)$ & $6(85.7 \%)$ & & \\
AA & & & & \\
GG & $3(10.7 \%)$ & $0(0 \%)$ & & \\
M3 /G857 & $0(0 \%)$ & $1(14.3 \%)$ & & \\
GA & $25(89.3 \%)$ & $6(85.7 .9 \%)$ & & \\
AA & & & \\
GG & & & \\
\hline
\end{tabular}

Table 5. Statistical comparison of NAT2 acetylation status between the study groups (group I, group II and group III), different stages and grades of bladder cancer using chi-square test

\begin{tabular}{|c|c|c|c|c|}
\hline & $\begin{array}{c}\text { Fast } \\
\text { acetylators }\end{array}$ & $\begin{array}{c}\text { Slow } \\
\text { acetylators }\end{array}$ & $\chi^{2}$ & $P$ \\
\hline \multicolumn{5}{|l|}{ Study groups } \\
\hline Group I (n=35) & 32 (93.3\%) & $3(6.7 \%)$ & \multirow{3}{*}{0.08} & \multirow{3}{*}{$>0.05$} \\
\hline Group II $(n=15)$ & $14(93.3 \%)$ & $1(6.7 \%)$ & & \\
\hline Group III (n=15 & 14 (93.3\%) & $1(6.7 \%)$ & & \\
\hline \multicolumn{5}{|l|}{ Tumor stages } \\
\hline Stage $1(n=17)$ & 14 (82.4\%) & 3 (17.6\%) & \multirow{3}{*}{3.54} & \multirow{3}{*}{$>0.05$} \\
\hline Stage $2(n=11)$ & 11 (100\%) & $0(0 \%)$ & & \\
\hline Stage $3(n=7)$ & 7 (11\%) & $0(0 \%)$ & & \\
\hline \multicolumn{5}{|l|}{ Tumor grades } \\
\hline Grade I $(n=28)$ & 25 (98.3\%) & $3(10.7 \%)$ & \multirow{2}{*}{0.82} & \multirow{2}{*}{$>0.05$} \\
\hline Grade II $(n=7)$ & $7(100 \%)$ & $0(0 \%)$ & & \\
\hline
\end{tabular}

by Nasr et al (12) to show the association between NAT2 polymorphism and bladder cancer in a group of patients from Lebanon. The study included 100 controls and 100 bladder cancer cases. Results showed no association between bladder cancer and slow/fast acetylator phenotypes of NAT2 in comparison to controls. Additionally, another study restricted to the United States done by Dong and Zhu (13) revealed no statistically significant increase in risk.

Yet the literatures are sometimes conflicting as a study conducted by Kalokairinou et al (5) for the relation of NAT2 gene polymorphism in addition to other risk factors such as age, gender and smoking with bladder cancer on Slovak population. The study included 90 bladder cancer cases and 274 healthy controls matched by ethnicity. It showed that NAT2 slow acetylator phenotype was significantly associated with bladder cancer risk. Another study was done by Hosen et al (1) that revealed a statistical significance of NAT2 slow acetylators with bladder cancer risk in Bangladeshi population.

On studying the effect of NAT2 genotypes and smoking on the risk of bladder cancer in comparison to the control groups, our results revealed no statistically significant difference between slow phenotype and smoking with bladder cancer. This could be explained by the high frequency of fast acetylators in our study. These results are in agreement with Mittal et al too (11).

In this study, the association of NAT2 phenotypes with the aggressiveness of bladder cancer using the different pathological tumor stages and grades were done. It revealed no statistical significant difference. This study goes, hand in hand with the study done by Mittal et al (11). On the other hand, Hosen et al (1) showed that patients with slow genotypes have high-grade tumor than those with fast genotypes.

Thus according to the above data, studies for NAT2 and bladder cancer risk are still inconclusive. The most important explanation for this discrepancy could be the high variability in the distribution of NAT2 alleles among the different populations. Hosen et al (1) reported that the slow alleles are predominated in $40 \%-60 \%$ in Caucasians including Indians, $5-25 \%$ in East Asians, $74 \%$ in South Indians and $21 \%$ in Bangladeshi subjects. In this study, we found, the low frequency of slow acetylators in our studied groups (8.6\% in bladder cancer patients and $6.7 \%$ in each control group). However, further studies with large sample size are needed.

The discrepancy of results may also be explained by the variable exposure to aromatic amines between different regions, including smoking habits or occupational exposure which may be a contributing factor (14). In addition to the potential role of other genetic factors such as NAT1 or GSTM1 (glutathione S-transferase mu1 class) while, GSTM1 null genotype increases the overall risk of bladder cancer (15), or, NAT1gene polymorphism especially NAT ${ }^{*} 10$ allele which is responsible for the higher levels of metabolic activation of N-hydroxyaromatic amines in human urinary bladder cytosol and human uroepithelial cells (16).

\section{Conclusion}

In conclusion, this investigation signifies that NAT2 genotype exhibit no relationship with the risk of growing bladder cancer, either alone or with smoking. Moreover, there was no association between NAT2 polymorphism and different stages and grades of bladder cancer.

\section{Limitations of the study}

The relatively small sample size of this study is considered as a limitation. We suggest conducting this investigation on a larger sample of bladder cancer patients. 


\section{Authors' contribution}

SAR supervised, reviewed and validated the final manuscript and was responsible for conceptualization. $\mathrm{HMZ}$ and $\mathrm{SAB}$ both of them contributed to the writing and editing of the manuscript and contributed to setting the research methodology, while MK contributed to the manuscript review and sample collection. Finally, WIH was responsible for original draft preparation and writing, sample collection and research methodology. All authors read, revised and approved the final manuscript.

\section{Ethical considerations}

Ethical issues (including plagiarism, data fabrication, double publication) have been completely observed by the authors.

\section{Conflicts of interest}

The authors declare no conflict of interest.

\section{Funding/Support}

No sources of funding obtained to support the study.

\section{References}

1. Hosen MB, Islam J, Salam MA, Islam MF, Hawlader MZ, Kabir Y. N-acetyltransferase 2 gene polymorphhism as a biomarker for susceptibility to bladder cancer in Bangladeshi population. Asia Pac J Clin Oncol. 2015; 11: 78-84. doi: 10.1111/ajco.12291.

2. Cui X, Lu X, Hiura M, Omori H, Miyazaki W, Katoh T. Association of genotypes of carcinogen-metabolizing enzymes and smoking status with bladder cancer in a Japanese population. Environ Health Prev Med. 2013;18: 136-142. doi: 10.1007/s12199-012-0302-x.

3. El-Sharkawi F, El Sabah M, Hassan Z, Khaled H. The biochemical value of urinary metalloproteinases 3 and 9 in diagnosis and prognosis of bladder cancer in Egypt. J Biomed Sci. 2014; 21:72-79. doi: 10.1186/s12929-014-00724.

4. Wu K, Wang X, Xie Z, Liu Z, Lu Y. N-acetyltransferase1 polymorphism and bladder cancer susceptibility: a metaanalysis of epidemiological studies. J Int Med Res. 2013;41: 31-37. doi: 10.1177/0300060513476988

5. Kalokairinou K, Ploumidis A, Kalogeropoulos T, Vlachos L, Stringaris K, Tavernaraki A, et al. The role of virtual cystoscopy, after multidetector computed tomography imaging reconstruction without the use of contrast medium, in the diagnosis and evaluations of bladder tumors: Preliminary Study. Adv Urol. 2014;146:222-240. doi:
$10.1155 / 2014 / 923958$

6. Vilčková M, Jurečeková J, Dobrota D, Habalová V, Klimčáková L, Waczulíková I, et al. Variation in $\mathrm{N}$-acetyltransferase 2 (NAT2), smoking and risk of prostate cancer in the Slovak population. Med Oncol. 2014;31:987. doi: $\quad 10.1007 / \mathrm{s} 12032-014-0987-3$.

7. Kamel A, Ebid G, Moussa H. N-Acetyltransferase 2 (NAT2) polymorphism as a risk modifier of susceptibility to pediatric acute lymphoblastic leukemia. Tumour Biol. 2015; 36:6341-8. doi: 10.1007/s13277-015-3320-7.

8. Wang C, Ross W, Mysorekar I. Urothelial generation and regeneration in development, injury, and cancer. Dev Dyn. 2017;246:336-43. doi:10.1002/dvdy.24487.

9. Xu X, Mathieu C, Berthelet J, Duval R, Bui LC, Busi F, et al. Human arylamine $n$-acetyltransferase 1 is inhibited by the dithiocarbamate pesticide thiram. Mol Pharmacol. 2017; 92: 358-65. doi:10.1124/mol.117.108662.

10. Zhao M, He XL, Teng XD. Understanding the molecular pathogenesis and prognostics of bladder cancer. Chin J Cancer Res. 2016;28:92-8. doi: 10.3978/j.issn.10009604.2016.02.05.

11. Mittal RD, Srivastava DS, Mandhani A. NAT2 gene polymorphism in bladder cancer: A Study from North India. Int Braz J Urol. 2004;30:279-88. doi: 10.1590/S167755382004000400003.

12. Nasr R, Temraz S, Mukherji D, Shamseddine A, Akika $\mathrm{R}$, Abbasi S, et al. Distribution and role of genetic polymorphisms of $\mathrm{N}$-acetyl tranferase2 (NAT2) enzyme in bladder cancer risk in the Lebanese population. Asian Pac J Cancer Prev. 2017;18:2561-8. doi:10.22034/ APJCP.2017.18.9.2561.

13. Dong Z, Zhu H. Single-strand conformational polymorphism analysis: basic principles and routine practice. Methods Mol Med. 2005;108:149-157.

14. Guaoua S, Ratbi I, Laarabi FZ, Elalaoui SC, Jaouad IC, Barkat A, et al. Distribution of allelic and genotypic frequencies of NAT2 and CYP2E1 variants in Moroccan population. BMC Genet. 2014;15: 156-160. doi: 10.1186/s12863-014-0156-X

15. García-Closas M, Malats N, Silverman D, Dosemeci M, Kogevinas M, Hein DW, et al. NAT2 slow acetylation, GSTM1 null genotype, and risk of bladder cancer: results from the Spanish Bladder Cancer Study and metaanalyses. Lancet. 2005;366:649-659. doi:10.1016/S01406736(05)67137-1.

16. Matic MG, Coric VM, Savic-Radojevic AR, Bulat PV, Pljesa-Ercegovac MS, Dragicevic DP, et al. Does occupational exposure to solvents and pesticides in association with glutathione S-transferase A1, M1, P1, and T1 polymorphisms increase the risk of bladder cancer? The Belgrade case-control study. PLoS One. 2014;9:488-99. doi: 10.1371/journal.pone.0099448.

Copyright $\odot 2019$ The Author(s); Published by Published by Society of Diabetic Nephropathy Prevention. This is an open-access article distributed under the terms of the Creative Commons Attribution License (http://creativecommons.org/licenses/by/4.0), which permits unrestricted use, distribution, and reproduction in any medium, provided the original work is properly cited. 